\title{
Segregación en las metrópolis españolas 2001-2011: un análisis con detalle territorial*
}

\author{
Miguel Rubiales Pérez
}

Universitat de Barcelona. Departamento de Geografía

mrubiales@ub.edu

\section{Resumen}

Durante la década que comprende los años 2001 y 2011 se producen cambios de gran calado en la sociedad y en las ciudades españolas: asistimos al auge y a la caída de una gran burbuja inmobiliaria, la composición poblacional se transforma en base a unos años de fuerte inmigración internacional y se produce un importante desarrollo de la suburbanización en las grandes urbes. ¿Han afectado estas transformaciones a la tradicionalmente estable distribución socioterritorial de la población? El análisis sustantivo de la segregación necesita detalle territorial porque se produce en grandes metrópolis compuestas por numerosos núcleos que son densos, compactos y heterogéneos. Los resultados ponen de relieve un tipo específico de polarización basada en la fragmentación de los espacios desfavorecidos. Solo desde una escala detallada podríamos evaluar el impacto de las políticas de intervención urbana en la fragmentación. Junto a métricas clásicas de la segregación, se emplean y se expanden las posibilidades de los indicadores locales de autocorrelación espacial (LISA) para dar cuenta de los cambios en la distribución socioterritorial de las regiones metropolitanas de Barcelona y Madrid durante el periodo 2001-2011. Los grupos poblacionales tomados en consideración incluyen diferentes ejes de jerarquización: categorías ocupacionales (directivos, técnicos y profesionales o trabajadores cualificados); país de nacimiento; formación universitaria, y tamaño de la vivienda.

Palabras clave: segregación; regiones metropolitanas; censo 2001-2011; clases sociales; población extranjera

* Este texto se enmarca en el proyecto I+D+I Nuevas movilidades y reconfiguración sociorresidencial en la poscrisis: Consecuencias socioeconómicas y demográficas en las áreas urbanas españolas (RTI2018-095667-B-I00), financiado por la Agencia Estatal de Investigación y el Fondo Europeo de Desarrollo Regional (AEI/ FEDER, UE). 
Resum. Segregació a les metròpolis espanyoles 2001-2011: una anàlisi amb detall territorial

Al llarg de la dècada compresa entre els anys 2001 i 2011 es produeixen grans canvis a la societat i a les ciutats espanyoles: s'assisteix a l'auge i caiguda d'una gran bombolla immobiliària, la composició per país d'origen es transforma a causa d'uns anys de forta immigració internacional, i se segueix produint un important desenvolupament de la suburbanització en les grans urbs. Han afectat aquestes transformacions a la tradicionalment estable distribució socioterritorial de la població? L'anàlisi substantiva de la segregació necessita d'un bon detall territorial, ja que es produeix en grans metròpolis compostes per nombrosos nuclis, que són densos, compactes i heterogenis. Els resultats posen de relleu l'existència d'una polarització específica, basada en la fragmentació dels espais més desafavorits. Només des d'una escala geogràfica detallada es podrà avaluar l'impacte de les polítiques d'intervenció urbana en la fragmentació. Al costat de les mètriques clàssiques de la segregació, s'utilitzen les possibilitats dels indicadors locals d'autocorrelació espacial (LISA) per retre compte dels canvis en la distribució socioterritorial de les regions metropolitanes de Barcelona i Madrid durant el període 2001-2011. Els grups poblacionals que s'han considerat inclouen diferents eixos de jerarquització: categories ocupacionals (directius, tècnics i professionals o treballadors qualificats); país de naixement; formació universitària, i grandària de l'habitatge.

Paraules clau: segregació; regions metropolitanes; cens 2001-2011; classes socials; població estrangera

Résumé. La ségrégation dans la métropole espagnole 2001-2011 : une analyse avec un détail territorial

La décennie comprise entre 2001 et 2011 a entraîné des changements majeurs dans la société et dans les villes espagnoles : nous assistons à la montée puisà la chute d'une grande bulle immobilière, la composition espagnole est transformée par des années de forte immigration internationale et un fort développement de la sous urbanisation dans les grandes villes. Ces transformations ont-elles affecté la répartition socio-territoriale traditionnellement stable de la population? Le recensement de 2011, clé pour apporter une réponse quantitative, a été réalisé sur un large échantillon. Ceci, qui n’est pas un problème pour les données agrégées, l'est pour une analyse territoriale détaillée. L'analyse de fond de la ségrégation nécessite des détails territoriaux car elle se produit dans de grandes métropoles composées de nombreux noyaux denses, compacts et hétérogènes. Nos résultats mettent en évidence un type de polarisation spécifique basé sur la fragmentation d'espaces défavorisés. Ce n'est qu'à cette échelle détaillée que nous obtenons une base pour évaluer l'impact des politiques d'intervention urbaine sur la fragmentation. Outre les mesures classiques de la ségrégation, les possibilités d'indicateurs d'autocorrélation spatiale locale (LISA) sont utilisées et étendues pour tenir compte de l'évolution de la répartition socio-territoriale des régions métropolitaines de Barcelone et de Madrid au cours de la période 2001-2011. Les groupes de population étudiés comprennent différents axes hiérarchiques: catégories professionnelles (gestionnaires et techniciens et professionnels ou travailleurs qualifiés); pays de naissance; formation universitaire, et taille du logement.

Mots-clés: groupes de population; régions métropolitaines; recensement 2001-2011; structure socio-territoriale; population étrangère

Abstract. Segregation in the Spanish metropolis 2001-2011: A detailed territorial analysis

Over the decade 2001 to 2011, major changes occurred in Spanish society and cities: the rise and fall of a large real estate market, population transformations following years of 
large-scale international immigration and the strong development of suburbanization. Have these transformations affected the traditionally stable socio-territorial distribution of the population? The 2011 census, key to a quantitative response, uses a large sample instead of a universal approach. This is not an issue problem for aggregate data, but does pose a problem for a detailed territorial analysis. A substantive analysis of segregation requires such territorial detail because it occurs in large metropolitan areas composed of numerous dense, compact and heterogeneous nuclei. Our results highlight a specific type of polarization based on the fragmentation of disadvantaged spaces. Only at this detailed scale can we obtain a basis for evaluating the impact of urban intervention policies on fragmentation. In addition to the classical segregation metrics, local spatial autocorrelation indicators (LISA) allow us explain changes in the socio-territorial distribution of the metropolitan regions of Barcelona and Madrid during the period 2001-2011. We analyze four population groups that serve as a basis to establish hierarchical relations of inequality: occupational categories (managers and technicians and professionals or skilled workers), country of birth, higher education, and size of residence.

Keywords: population; metropolitan regions; census 2001-2011; socio-territorial structure; foreign

\author{
Sumario \\ 1. Introducción 3. Análisis de los resultados \\ 2. Metodología 4. Conclusiones \\ Referencias bibliográficas
}

\title{
1. Introducción
}

En las últimas décadas, el interés (y la literatura) sobre segregación ha ido sustituyendo a la que se producía sobre desigualdades, especialmente en las aproximaciones cuantitativas (puede comprobarse en revisiones bibliográficas exhaustivas e internacionales: Lloyd y Shuttleworth, 2014; Sienkiewicz et al., 2016; Israel y Frenkel, 2018). La segregación, la separación de diferentes grupos en el espacio metropolitano, tiene importantes consecuencias sociales. Desde un punto de vista teórico, la relación de la segregación con las desigualdades no es directa, ni evidente, aunque muchos autores asimilen analizar segregación con dar cuenta de las desigualdades. Con frecuencia, esta asociación se justifica en base a un supuesto efecto barrio (Cox, 1969; Miller, 1977; Huckfeldt et al., 2000) que afectaría al logro escolar (Andersson y Malmberg, 2015), a la composición étnica (Clark et al., 2015) y la satisfacción vital (Knies et al., 2016). Pero este efecto barrio es cada vez más discutido (Sampson, 2019; Sharkey y Faber, 2014), especialmente en contextos diferentes del de Estados Unidos (Arbaci y Rae, 2014; Tapada-Berteli y Arbaci, 2011).

El análisis de las concentraciones socioespaciales se enriquece si, además, se atiende a las dinámicas de cambio (Tammaru et al., 2017) y a la otra dimensión de la segregación: la estigmatización territorial (Wacquant et al., 2014). Este 
concepto ayuda a comprender la formación de guetos y de «áreas sensibles» como parte de un proceso que va más allá de la concentración de la población con pocos recursos económicos, culturales y simbólicos. A pesar de esto, hay pocos trabajos que desarrollen cuantificaciones o indicadores alrededor de esta idea.

Cuantificar y cartografiar los cambios en la segregación es, a la luz de los datos disponibles, una tarea con dificultades metodológicas, pero resulta cada vez más necesaria en tanto el territorio adquiere un papel crecientemente importante en la propia estructura social del capitalismo avanzado (Harvey, 1998). El análisis de la segregación es necesario para hacer una valoración completa de las tendencias de las desigualdades de nuestra sociedad. Es también la base para saber en qué medida se está garantizando el derecho a la ciudad (Lefebvre, 1976; Harvey, 2008; Blokland et al., 2015) y resulta un paso imprescindible para valorar los impactos que están teniendo las políticas sectoriales y urbanísticas, tanto a escala individual como a escalas metropolitana y de ciudad. Precisamente ese es el objetivo del presente artículo: conocer cómo se han transformado la segregación residencial y las desigualdades socioterritoriales de las grandes metrópolis españolas con el máximo detalle territorial. Tammaru et al. (2017), analizando las dinámicas de la desigualdad territorializada en las regiones metropolitanas europeas, y López-Gay (2018) para Barcelona ${ }^{1}$, concluyen que se ha producido un aumento de la segregación y de la polarización. Los resultados de nuestro análisis también confirman esta línea de conclusiones y permiten tanto avanzar detalles sobre la forma de esta polarización como plantear la hipótesis de su posible relación con políticas urbanas dirigidas a la desconcentración de los grupos más vulnerables².

\section{Metodología}

El análisis se realiza sobre los datos de los censos de 2001 y 2011, a escala de secciones censales, para las regiones metropolitanas de Barcelona ${ }^{3}$ y Madrid ${ }^{4}$. Los grupos poblacionales tomados en consideración incluyen categorías ocupacionales (directivos, técnicos y profesionales o trabajadores cualificados), el país de nacimiento y la formación universitaria. Estas categorías son las que mejor detectan las desigualdades sociales en el territorio, como se ha comprobado en trabajos anteriores (Rubiales et al., 2012).

El núcleo de las cuantificaciones que se emplean descansa sobre los indicadores locales de autocorrelación espacial (LISA). Generalmente, la aplicación de los LISA en ciencias sociales se ha dirigido a la localización de concentraciones estadísticamente significativas de grupos sociales en la ciudad (Anselin,

1. De alguna forma, también Sorando y Leal (2019) para Madrid y Barcelona.

2. Este artículo desarrolla elementos parciales de una investigación mayor. Si el lector tiene interés en extenderse sobre los fundamentos teóricos, puede encontrarlos en Rubiales (2017a).

3. RMB, considerando la zona en la que proporciona servicio la entidad metropolitana del transporte.

4. Considerando la provincia de Madrid. 
1995; Esteve, 2003; Frank, 2003; Bayona y Blanco, 2004; Dawkins, 2004; Martori y Hoberg, 2008; Koch y Mandl, 2011). Su empleo como indicador agregado de segregación con datos del censo de 2011 resulta más reciente (Rubiales, 2017a, Sorando y Leal, 2019).

En este artículo se trabaja a nivel de secciones censales. Su utilización es de especial relevancia porque es la escala estadística más detallada, aquella en la que obtienen mayores valores en los índices de segregación, la que permite evitar las distorsiones de agrupación en barrios y distritos y la que daría pie a conectar los resultados con las intervenciones de políticas urbanas 5 . Las secciones censales son capaces de mostrar tanto la heterogeneidad interna de barrios y municipios como la cohesión y la continuidad en formaciones supramunicipales que agrupan un gran número de unidades vecinas. Esta decisión sobre la escala implica asumir un mayor error muestral de los datos, aunque matizado por la agrupación de categorías que se ha realizado.

La aproximación cuantitativa a los cambios en la segregación presenta varios problemas. En diez años se han producido variaciones en todos y cada uno de los elementos que deberían mantenerse constantes para dar cuenta cabal de las transformaciones. En primer lugar, los procesos oficiales de recuento han cambiado: el censo ha pasado de ser universal a ser muestral y se han redibujado las delimitaciones de las secciones censales. En segundo lugar, hay importantes variaciones en la distribución de las poblaciones, sus totales y sus pesos relativos como resultado de tres grandes procesos: el fuerte aumento de la población extranjera (Domingo, 2014; Bayona y Gil, 2012), el desarrollo de la suburbanización metropolitana (Champion, 2001; Coll y Pujadas, 2007; Pujadas, 2009) y el deterioro sociolaboral relacionado con la crisis iniciada en 2009 (Rubiales et al., 2013; Colectivo IOÉ, 2012; Rocha y Aragón, 2012). Por último, muchas categorías podrían no ser exactamente comparables consigo mismas a causa de procesos de reclasificación oficiales, como la nueva clasificación de ocupaciones de 2010, o en algunos casos por haber sufrido intensas transformaciones de su composición interna. Por ejemplo, la composición por

5. Hay un matiz muy interesante que diferencia la escala a la que se obtienen mayores valores de segregación con la escala a la que se producen los mayores niveles de la segregación (Manley et al., 2019). Este detalle resulta de especial relevancia para los estudios sobre segregación étnica. Es frecuente que los colectivos muy minoritarios, como tiende a ocurrir al inicio de la relación migratoria, se concentren en una única zona, como las diferentes Chinatowns. En este caso, la presencia de un grupo en un único distrito es la mayor responsable de su separación respecto al resto de la población. A pesar de esto, conforme se calcula la segregación a escalas más detalladas, los valores aumentan ligeramente. Las escalas más detalladas incorporan el nivel de segregación presente en la anterior y le añaden el suyo propio. En nuestro ejemplo sería un aumento pequeño, porque el mayor salto se habría producido al pasar de la escala ciudades a la escala distritos. De esta forma, aunque los mayores valores de segregación siempre aparecen en las escalas más detalladas, no necesariamente se han producido a ese nivel. En todo caso, las metodologías monoescalares tienen un interés evidente en usar las escalas más detalladas. Los valores obtenidos empleando una escala mayor como las agrupaciones de secciones o los distritos no tienen en cuenta la segregación de las escalas más detalladas, pero lo contrario no ocurre y los valores obtenidos con la escala de sección censal sí que incorporan el efecto de la segregación producida en los niveles superiores. 
países de la población extranjera de 2001 es diferente a la de 2011, especialmente en la ciudad de Barcelona. Sin poder resolver estos problemas, los datos se analizan mediante cuatro estrategias complementarias:

1. El establecimiento de cuantificaciones clásicas de índices de segregación: la distancia media $\left(P_{x x}\right)$ que mide la concentración, el propio índice de segregación (IS), que es una medida global de igualdad y concentración de la distribución, y los cocientes de localización $(L Q)$, que funcionan como medidas locales de concentración y solucionan el problema de localizar y comparar con precisión diferentes concentraciones relativas:

$$
\begin{gathered}
P_{x x}=\frac{1}{X^{2}} \sum_{i=1}^{n} \sum_{j=1}^{n} x_{i} x_{j} d_{i j} \\
I S=\frac{1}{2} \sum_{i=1}^{n}\left|\frac{x_{i}}{X}-\frac{t_{i}-x_{i}}{T-X}\right| \\
C L=\frac{\frac{x_{i}}{t_{i}}}{\frac{X}{T}}
\end{gathered}
$$

Donde:

n Número de unidades espaciales en el área metropolitana.

$T$ Población total en el área metropolitana.

$t_{i} \quad$ Población total en la unidad espacial $i$.

$X \quad$ Población total del grupo $X$ en el área metropolitana.

$x_{i} \quad$ Población total del grupo $X$ en la unidad espacial $i$.

$x_{j} \quad$ Población total del grupo $X$ en la unidad espacial $j$.

$\dot{d}_{i j} \quad$ Distancia del centroide de la unidad $i$ hasta el de la unidad.

2. La comparación de la evolución de los valores globales de autocorrelación (la $I$ de Moran), que indica si aumenta o no la concentración y la agrupación espacial de los grupos. El estadístico sobre la autocorrelación espacial más utilizado es la I de Moran (Moran, 1948):

$$
I=\frac{n \sum_{j}^{n}={ }_{1} W_{i, j} Z_{i} Z_{j}}{S_{0} \sum_{i=1}^{n} Z_{i}^{2}}
$$

Donde:

$Z_{i} \quad$ Es la desviación de la variable de interés con respecto a la media $\left(x_{i}-x\right)$.

$z_{i} \quad$ Es la diferencia respecto a la media en la unidad $i$.

$Z_{j} \quad$ Es la diferencia respecto al valor medio en la unidad $j$, vecina de $i$.

$W_{i, j}$ Es el valor de la matriz que indica si $i$ y $j$ son consideradas (1) o no vecinas (valor 0 ). 
$n \quad$ Es el número de unidades espaciales.

$S_{0} \quad$ Es el agregado, el número total, de todos los cruces entre vecinos, un sumatorio de las deviaciones cruzadas de todos los vecinos.

3. El detalle de los recuentos globales de los indicadores locales de autocorrelación que señalan, dentro de estos cambios generales, qué parte corresponde a variaciones en las zonas de concentración de poblaciones privilegiadas y qué parte a zonas de concentración de poblaciones desfavorecidas.

A partir de la $I$ de Moran, Anselin (1995) propone realizar los mismos test de hipótesis, pero para el valor Li de cada una de las unidades espaciales. De esta forma, se clasifican las unidades en cinco grupos, un primer grupo de aquellas unidades que no se desvían de los valores esperados (medios) y cuatro grupos en los que se producen desviaciones, bien porque la unidad y sus vecinas presentan valores muy por encima de la media, bien porque presentan valores muy por debajo de la media, o porque los valores de la unidad están por encima de la media y en sus vecinas por debajo, o porque los valores son especialmente bajos en la unidad y especialmente altos en las unidades vecinas (Anselin et al., 2006) ${ }^{6}$.

$$
I_{i}=\frac{z_{i} \sum_{j} w_{i, j} z_{j}}{\sum_{i} z_{i, j / n}^{2}}
$$

Donde:

$z_{j} \quad$ Es la diferencia respecto a la media en la unidad $i$.

$z_{i} \quad$ Es la diferencia respecto al valor medio en la unidad de $j$, vecina de $i$.

$W_{i, j}$ Es el valor de la matriz que indica si $i$ y $j$ son consideradas (1) o no vecinas (valor 0 ).

$n \quad$ Es el número de unidades espaciales.

Los LISA son una adaptación de los valores locales del índice de autocorrelación espacial de Moran para detectar patrones locales de asociación espacial. Al buscar asociación espacial funcionan, a la vez, como cuantificación de la igualdad y de la agrupación de la distribución de una población en el territorio. Estos indicadores ocupan un papel destacado en nuestro análisis, porque al hacer el contraste en función de su valor y del de sus vecinas, los LISA tienen en cuenta, a la vez, las dimensiones de concentración y de agrupación de la segregación en una dimensión híbrida que denominaremos a partir de ahora barrialización. Estas barrializaciones pueden tratarse como indicadores globales mediante el recuento de la proporción de población que reside en ellas. Así los LISA permiten obtener medidas

6. Para una ampliación del funcionamiento de esta cuantificación, puede ser ilustrativo consultar el trabajo de Folch y Rey (2016). Los autores valoran el potencial del índice de centralización como medida de segregación explícitamente espacial. Para ello, entre otras operaciones, comparan su funcionamiento con el de los indicadores locales de autocorrelación. 
no centrales de la autocorrelación espacial capaces de sacar a la luz la composición interna del indicador global:

- Denominaremos $A 2$ a las barrializaciones o zonas con concentraciones contiguas de valores significativamente altos según los LISA. Estas concentraciones pueden estar marcando una zona privilegiada (cuando se refieren a residentes en viviendas de más de $120 \mathrm{~m}^{2}$, trabajando en niveles laborales altos: directivos, técnicos científicos y profesionales o población universitaria) o una zona desfavorecida (cuando hacen referencia a la población extranjera). Existe su valor equivalente pero calculado a partir de la población residente en zonas cuyo cociente de localización duplica la concentración media ( $L Q A 2)$.

- Denominaremos $A 1 / 2$ a las barrializaciones o zonas con valores significativamente bajos, rodeadas de otras zonas con valores que también son bajos según los LISA. Su equivalencia en cocientes de localización es el $L Q A 1 / 2$ — valores inferiores a la mitad de la media-). De nuevo, estas zonas señalan privilegio o desventaja en función del grupo para el que se estén realizando los cálculos.

4. La representación cartográfica de los indicadores locales de autocorrelación para identificar los patrones morfológicos de las concentraciones, su ubicación metropolitana y el lugar donde se producen las principales transformaciones (ver la discusión de resultados y las figuras 2 y 3 ). Esta forma de evaluar las transformaciones permite valorar los cambios independientemente del rediseño de las secciones censales y sin recurrir a su agrupación.

\section{Análisis de los resultados}

\subsection{Evolución de cuantificaciones que aproximan segregación}

En la tabla 1 se presentan, para los años 2001 y 2011, varias cuantificaciones de la segregación: los valores globales de autocorrelación (tanto el general como los recuentos locales de barrializaciones $A 2$ y $A 1 / 2$ ), la distancia media de los miembros del grupo $(P x x)$, los cocientes de localización $(L Q)$ y el índice de segregación $(I S)$. Estas tres últimas medidas se emplean como complemento del análisis basado en la autocorrelación espacial. Todas las cuantificaciones se aplican a una selección de las categorías más directamente relacionadas con la jerarquización socioespacial: porcentaje de población en casas de más de 120 $\mathrm{m}^{2}$, en ocupaciones de categoría alta, con estudios universitarios y que tengan nacionalidad extranjera.

La distancia media de los miembros del grupo $(P x x)$ da cuenta de un proceso de expansión del conjunto de la población. Aumenta en términos generales un $4,76 \%$ (ver la tabla 1), aunque se presentan variaciones en función del grupo considerado: la población en ocupaciones de mayor categoría se dispersa al ritmo casi idéntico al del conjunto de la población (un 4,77\%). Hay grupos, la población residente en viviendas grandes y la población extranjera, 
Tabla 1. Valores y cambio porcentual en la distancia media entre miembros del grupo (Pxx), la autocorrelación espacial global y local y el índice de segregación en las RMB y RMM

\begin{tabular}{|c|c|c|c|c|c|c|c|c|c|c|c|c|c|}
\hline \multirow[b]{2}{*}{ Indicador } & \multirow{2}{*}{$\begin{array}{l}\text { Total } \\
\% \text { var. }\end{array}$} & \multicolumn{3}{|c|}{ 0cupaciones altas } & \multicolumn{3}{|c|}{ Viviendas $120 \mathrm{~m}^{2}$} & \multicolumn{3}{|c|}{ Población ext. } & \multicolumn{3}{|c|}{ Título universitario } \\
\hline & & 2001 & 2011 & $\%$ var. & 2001 & 2011 & $\%$ var. & 2001 & 2011 & $\%$ var. & 2001 & 2011 & $\%$ var. \\
\hline Pxx & 4,8 & 17,2 & 18,0 & 4,8 & 15,1 & 16,0 & 6,3 & 19,0 & 20,5 & 8,2 & 19,7 & 20,1 & 1,9 \\
\hline \multicolumn{2}{|l|}{ I de Moran } & 0,83 & 0,62 & $-24,64$ & 0,69 & 0,60 & $-12,88$ & 0,59 & 0,20 & $-65,53$ & 0,85 & 0,74 & $-12,68$ \\
\hline \multicolumn{2}{|c|}{ LISA -A2- } & 0,19 & 0,21 & 8,94 & 0,11 & 0,07 & $-37,17$ & 0,15 & 0,15 & $-0,46$ & 0,20 & 0,18 & $-9,61$ \\
\hline \multicolumn{2}{|c|}{ LISA -A1/2- } & 0,30 & 0,23 & $-21,22$ & 0,22 & 0,08 & $-61,54$ & 0,28 & 0,25 & $-12,60$ & 0,27 & 0,18 & $-32,20$ \\
\hline \multirow[t]{3}{*}{ IS } & & 0,23 & 0,30 & 27,79 & 0,42 & 0,45 & 7,45 & 0,30 & 0,38 & 25,65 & 0,16 & 0,27 & 64,23 \\
\hline & LQ 2 & 0,11 & 0,10 & $-3,89$ & 0,18 & 0,19 & 8,82 & 0,11 & 0,12 & 14,57 & 0,13 & 0,11 & $-17,28$ \\
\hline & LQ 1/2 & 0,26 & 0,31 & 17,03 & 0,56 & 0,57 & 0,90 & 0,30 & 0,33 & 10,88 & 0,35 & 0,27 & $-22,95$ \\
\hline
\end{tabular}

Fuente: elaboración propia a partir de dato del censo de 2001.

que se dispersan más que la media (un 6,27\% y un 8,21\%, respectivamente). $\mathrm{Y}$, finalmente, la población con estudios universitarios o superiores, que sufre un proceso de concentración relativa, con un incremento de la distancia media entre sus miembros solo del 1,89\%, muy por debajo del ritmo de dispersión general. Estos datos destacan la concentración en la capital de población universitaria y la dispersión metropolitana de la población extranjera.

\subsection{Evolución de las cuantificaciones de autocorrelación como valores globales}

La autocorrelación espacial, medida con la $I$ de Moran, presenta valores mayores para las variables de estudios y ocupaciones superiores (autocorrelaciones espaciales entre el 0,75 y el 0,85 ). La importancia de esta agrupación se reduce para la población residente en viviendas grandes (valores de autocorrelación espacial sobre el 0,6 ) y para la población extranjera (entre el 0,58 de 2001 y el 0,2 de 2011). Se comprueba que los cambios en la $I$ de Moran guardan relación con las diferencias relativas en los ritmos de dispersión $\left(P_{x x}\right)$. Se producen caídas generalizadas congruentes con un aumento general de la dispersión de la población, posiblemente asociada al desarrollo de la suburbanización. Cuanto más intenso es el proceso de dispersión de los miembros de un grupo, mayor es la reducción de la autocorrelación espacial para ese grupo. Esta relación se observa claramente en la mayor dispersión que sufre la población extranjera, que lleva a una gran caída de su autocorrelación espacial en un $60 \%$ entre 2001 y 2011. En el extremo opuesto, la población universitaria, que sufre un proceso de concentración relativa, es la que menos reduce su autocorrelación espacial, solo un 12,7\% (pasa del 0,85 al 0,74). Entre ambas se encuentra la población ocupada, que se dispersa al ritmo del conjunto, con una caída de la $I$ de Moran del 24\% (pasa del 0,83 al 0,62). Finalmente, la población residente en viviendas de más de $120 \mathrm{~m}^{2}$ mantiene valores más altos de $I$ de Moran que otras variables, porque, aunque se dispersa más que la media, presenta una caída muy moderada de los valores de autocorrelación, un 12,9\% (pasa del 0,69 al 0,60). 
Una parte de la caída en los valores generales de autocorrelación podría deberse a la reducción de la desigualdad de la distribución (ver la tabla 1), pero no parece ser el caso. Durante el periodo 2001-2011 el índice de segregación aumenta para las cuatro categorías estudiadas: la población ocupada en empleos de categoría alta, la residente en viviendas de más de $120 \mathrm{~m}^{2}$, la de nacionalidad extranjera y la población con estudios universitarios. Los estudios superiores, la variable que más incrementa la desigualdad de su distribución (de 1,62 a 2,67), es una de las que más crece durante el periodo, y lo hace de una forma más urbana y concentrada. Al contrario, la población residente en viviendas grandes constituye un grupo más reducido, con un ritmo lento de crecimiento, y se mantiene casi estancada en los niveles altos de segregación (pasa del 0,42 al 0,45$)$. La población extranjera y la de ocupaciones altas presentan subidas medias de la desigualdad en la distribución de una cuarta parte del valor inicial, con lo que se sitúan respectivamente con un $I S$ de 0,38 y de 0,30 en 2011.

Respecto al análisis de barrializaciones, valores $A 2$ y $A 1 / 2$, si consideramos la evolución de la población residente en estas zonas, vemos que la variación de la $I$ de Moran para la población ocupada en categorías altas no es internamente homogénea. Mientras las unidades administrativas donde no viven gerentes ni profesionales liberales pierden continuidad y pasan a estar menos agrupadas (reducen su $A 1 / 2$ de un 29,5 a albergar solo un 23,2\% del total), el número de habitantes de secciones donde se concentra la población de ocupaciones altas (A2) aumenta de un 18,9\% hasta el 20,5\% del total, con lo que se barrializan más. Se produce una diferenciación similar si consideramos la igualdad de la distribución, medida en las secciones con valores de $L Q$ extremos. En 2001 había 825 secciones censales en las que la población ocupada en las categorías altas sobrepasaba la media ( $L Q A 2)$ y albergaban un $10,53 \%$ de la población total. Diez años más tarde, en 2011, este porcentaje se reduce muy ligeramente, hasta el 10,12\% del total de la población (en 817 secciones censales). Esta ligera reducción en los $L Q A 2$ contrasta con el aumento de la población (del 26,4\% al 30,8\% del total) en los $L Q A 1 / 2$, lo que podría indicar una mayor concentración de pobreza en las secciones desfavorecidas. Medidas con una lógica diferente, ofrecen resultados consistentes: parece que los barrios favorecidos se extienden y mantienen su alta proporción de población en ocupaciones altas, mientras que los barrios desfavorecidos pierden extensión continua. Esto no quiere decir que se reduzca su segregación, puesto que estos fragmentos de población desfavorecida forman cada vez menos barrios compactos, se fragmentan, pero dentro de esos fragmentos la proporción de población desfavorecida que reside en ellos se concentra aún más.

\subsubsection{Población universitaria}

Respecto a la población universitaria, se observa un aumento de la desigualdad y un proceso de generalización que reduce los valores de agrupación y la concentración. Se reducen tanto las poblaciones residentes en secciones con alta autocorrelación espacial (del $20 \%$ y el $18,08 \%$ del total para $A 2$ y del $26,74 \%$ al $18,13 \%$ del total para $A 1 / 2$ ) como las residentes en secciones con 
$L Q$ alejados de la media (caída del $13,27 \%$ al 10,98\% para $L Q A 2$ y del 34,5\% al $26,58 \%$ para $L Q A 1 / 2$, valores de la mitad o menos de la media). Así, las mayores reducciones se producen respecto a las secciones con ausencia de población universitaria, que de 2001 a 2011 pasan a tener más personas licenciadas viviendo en ellas. Una posible explicación sería que mientras el relevo generacional en las áreas favorecidas de la ciudad implica una reproducción de la proporción (alta) de población con título universitario, en las zonas desfavorecidas este relevo (tanto si es de personas extranjeras como si no) supone un aumento de la proporción que tiene educación superior.

El cambio en la distribución de la población residente en viviendas de más de $120 \mathrm{~m}^{2}$, durante el periodo de 2001 a 2011 (ver la tabla 1), se parece más a las transformaciones en la distribución de ocupaciones altas que a la democratización de los estudios superiores en las zonas urbanas densas. En primer lugar, descienden los valores de agrupación, pero se desagrupan con mucha más intensidad las zonas $A 1 / 2$ (pasan del 21,7\% al 8,3\%) que las $A 2$ (del 11\% al 7\%). Los cocientes de localización permiten afirmar que esta desagrupación no está relacionada con una reducción de la desigualdad. Los valores extremos de $L Q$ aumentan en las secciones en las que la proporción de directivos y profesionales liberales duplica el porcentaje medio (estos $L Q$ superiores a 2 pasan del $17 \%$ al $19 \%$ de la población total), mientras que los valores de $L Q$ inferiores $(A 1 / 2)$ se mantienen estables (en torno al $56 \%$ de la población). Estos datos son congruentes con el crecimiento de las zonas de viviendas grandes en forma de mancha de aceite y con un aumento generalizado de las viviendas de tamaño medio, tanto por nueva creación como por reforma de viviendas más pequeñas.

\subsubsection{Población extranjera}

La gran caída en los valores de autocorrelación de la población extranjera no significa que esta población haya aumentado su integración espacial. A pesar de su desconcentración y su gran aumento poblacional entre 2001 y 2011, los recuentos poblacionales a partir de valores LISA y $L Q$ no sufren caídas generalizadas, puesto que solo se reducen los valores de $A 1 / 2$ (del $28,1 \%$ al 24,6\%). En el otro extremo la agrupación de las secciones censales con fuerte concentración de extranjeros $(A 2)$, los valores de barrialización se mantienen estables (un 14,9\% de la población en 2001 y en 2011). Es decir, la población extranjera mantiene su distribución desigual y su concentración, pero se ha extendido también por nuevas zonas. Lo que sí ha ocurrido con claridad ha sido un aumento en los extremos de su distribución. La población residente en secciones con valores altos de $L Q$ se incrementa (del 10,6\% al 12,1\%).

\subsubsection{Ocupaciones y clases}

La desbarrialización de la población en función de la ocupación tiene un sesgo central (ver la figura 1). Respecto a su desequilibrio interno, en las ciudades de Madrid y Barcelona es donde más crece la barrialización de las $L Q$ ocupacionales altas y en las regiones metropolitanas, donde más desaparece la barriali- 
zación desfavorecida. El aumento de la desigualdad presenta cierto sesgo hacia las periferias trabajadoras. Generalmente, las diferencias entre Madrid y Barcelona se deben a los municipios obreros conurbados e incluidos en el término municipal de Madrid. Así, tanto en función del IS global como de los valores de los $L Q$ bajos, la ciudad de Madrid y la RMB aumentan más sus desigualdades que la provincia de Madrid y la ciudad de Barcelona, respectivamente. Por el contrario, el aumento de la desigualdad derivado de un incremento en las zonas con ocupaciones altas (LQA2) tiene un sesgo más central (tanto en Barcelona como en Madrid). Estos resultados son congruentes con procesos de elitización de los centros.

La desagrupación de los barrios con inmigrantes (ver la figura 1) es también un fenómeno de ámbito central. En las capitales es donde más se produce una difusión generalizada, que implica la reducción tanto de los barrios relativamente llenos $(A 2)$ como los que están vacíos de población extranjera $(A 1 / 2)$. Sin embargo, en las regiones metropolitanas, las barrializaciones, la agrupación de secciones con niveles altos de población extranjera, aumenta. Este cambio podría está dando cuenta de procesos de desplazamiento de los barrios de concentración de la población extranjera hacia segundas y terceras coronas metropolitanas. Es una dinámica de cambios congruentes con los datos sobre igualdad en la distribución. Las zonas de concentración ( $L Q A 2)$ de población extranjera se reducen más, o crecen menos, en las ciudades centrales. Mientras tanto, las zonas relativamente vacías de inmigrantes ( $L Q A 1 / 2)$ crecen más en la región metropolitana de Barcelona. En resumen, las tendencias de difusión general y reducción de barrios de concentración sí ocurren en las capitales, pero no en las regiones metropolitanas. Este cambio puede estar relacionado con un mayor atractivo de las zonas de periferia para la población extranjera, en parte por dinámicas de desplazamiento de esta población hacia las segundas y terceras coronas. En menor medida, también hay que tener en cuenta el proceso de nacionalización diferencial de la población latinoamericana, que se asienta preferentemente en las capitales.

\subsubsection{Residentes en viviendas de más de $120 \mathrm{~m}^{2}$}

$\mathrm{Al}$ analizar el cambio de la población residente en viviendas de más de $120 \mathrm{~m}^{2}$ (ver la figura 1) aparecen diferencias regionales sistemáticas entre Barcelona y Madrid. Las caídas de la $I$ de Moran y los LISA son más pronunciadas en Madrid capital y en la RMB que en el resto de Madrid y en Barcelona capital. Esto indica que la reducción de desigualdad y agrupación se produce especialmente en los cinturones obreros que forman la primera corona de Barcelona y la zona sur del municipio de Madrid. Si hubiera que aventurar una hipótesis coherente con estos datos, sería que en estas zonas ha aumentado a mayor velocidad la población con viviendas grandes, aunque no dejaría de ser una especulación. Finalmente, las diferencias entre ámbitos del cambio en los indicadores sobre población universitaria (G3, ver figura 1) son compatibles con un proceso de difusión generalizada sin evidentes diferencias en función de los ámbitos. 
Figura 1. Variación en valores de barrialización y $L Q$ para ocupación alta, viviendas de más de $120 \mathrm{~m}^{2}$, población extranjera y universitaria en diferentes ámbitos territoriales
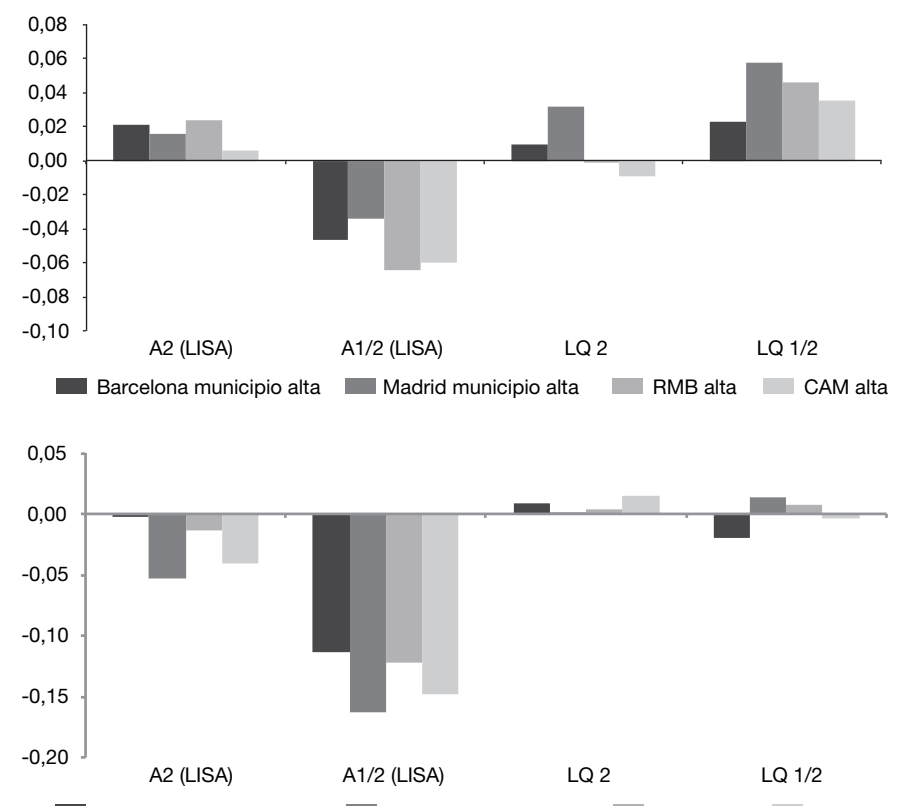

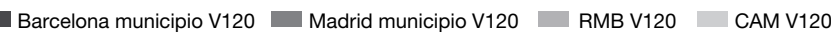
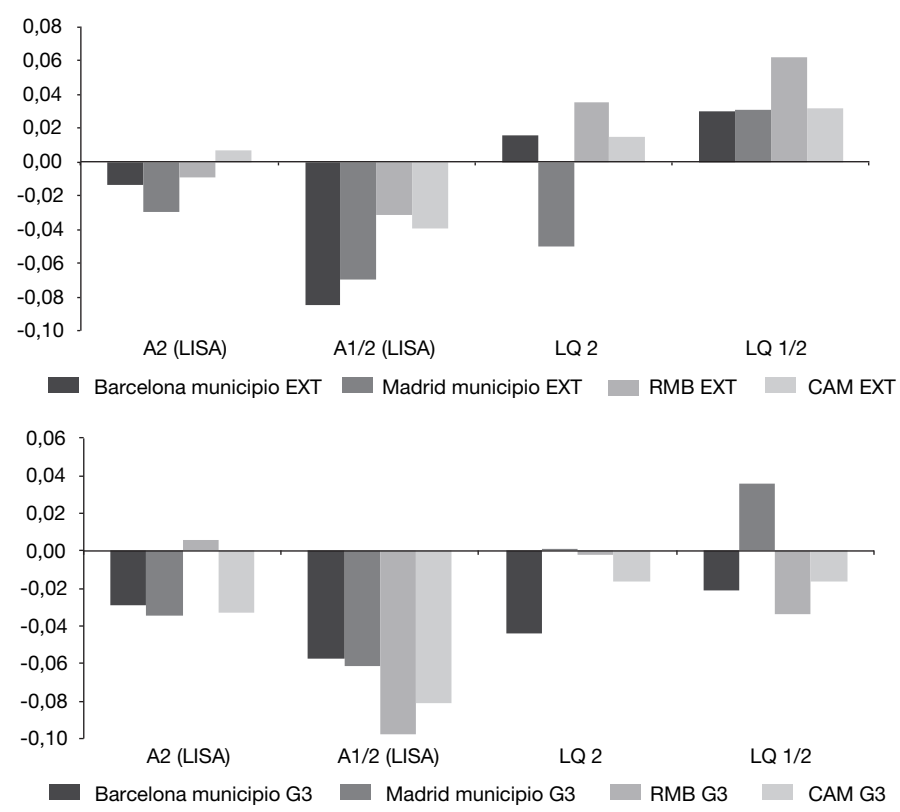

Fuente: elaboración propia a partir de datos del censo de 2001. 


\subsection{Representación cartográfica de los cambios en los LISA}

La representación cartográfica de las zonas de concentración y agrupación de un grupo en niveles estadísticamente significativos (barrializaciones) también permite formar una imagen de la interrelación entre diferentes variables y ejes de jerarquización socioespacial: población ocupada en categorías altas, residente en viviendas de más de $120 \mathrm{~m}^{2}$, de población extranjera y con estudios de tercer ciclo completados. Además, también permite dar cuenta de los cambios relativos producidos entre 2001 y 2011 . En las figuras 2 y 3 se muestran detalles de la cartografía comparativa entre barrializaciones (solo las $A 2$, las de concentración por razones de claridad en la representación cartográfica). Las barrializaciones de población residente en viviendas de más de $120 \mathrm{~m}^{2}$ se representan en una transparencia lila oscuro; en una transparencia azul, las referidas a la población que desempeña su labor profesional en ocupaciones altas; en rojo, las de población con título universitario. Cuando coinciden las tres, el color lila se intensifica marcando las zonas con población relativamente privilegiada. Las zonas representadas en amarillo representan barrializaciones A2 de población extranjera, relativamente desfavorecida.

La presencia de una zona de barrialización de un grupo - por ejemplo, personas con título universitario- no quiere decir que en ella las tituladas universitarias sean mayoría. También podría incluso ocurrir que la sección censal con mayor porcentaje de un grupo quedara excluida de la barrialización. Lo que sí se puede decir de las barrializaciones $(A 2)$ es que son espacios urbanos en los que ese grupo presenta fuertes concentraciones de forma continuada. Es el lugar de la ciudad donde, si alcanza cierta masa crítica, ese grupo es capaz de «hacer barrio» dejando sentir en él su impronta y dando pie a la asociación entre grupo social y territorio urbano.

La primera característica que se resalta en las figuras 2 y 3 es la superposición de capas. A modo de muñecas rusas, la barrialización $(A 2)$ de la población residente en viviendas grandes está incluida en la barrialización de población ocupada en categorías altas y, a su vez, esta está incluida en la barrialización de la población titulada universitaria. Las diferencias de extensión y localización dan pistas sobre la propia articulación interna de la clase alta en función de las dimensiones de patrimonio, renta y capital cultural institucionalizado. Así, la máxima extensión es la de la barrialización de la población con título universitario en 2011. La agrupación espacial de la población con capital cultural institucionalizado abarca casi la mitad de las ciudades centrales: los distritos de Les Corts, Sarrià-Sant Gervasi, Gràcia, Eixample y, parcialmente, Sant Martí (en Barcelona); Chamartín, Salamanca, Retiro, Moncloa y, parcialmente, Centro y Arganzuela (en Madrid).

\subsubsection{Categorías ocupacionales altas}

Un grupo más restringido espacial y socialmente es el de la población con ocupaciones de categorías altas, que Moreno et al. (2003) demuestra fuertemente asociadas con la renta. La concentración y agrupación $(A 2)$ de la población con 
Figura 2. Barrializaciones superpuestas: vivienda de más de $120 \mathrm{~m}^{2}$, ocupación alta, formación universitaria y nacionalidad extranjera (Barcelona)
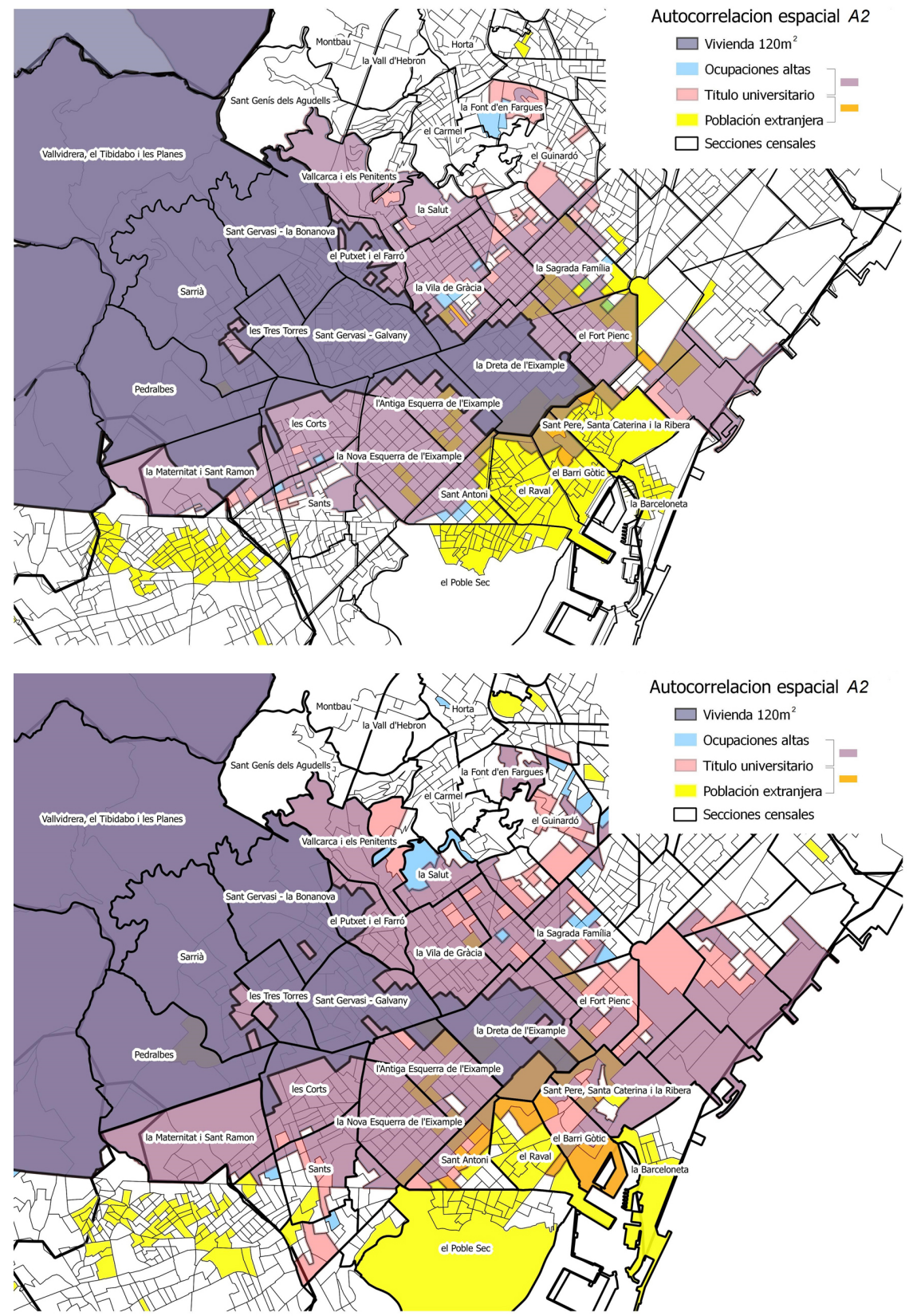

Fuente: elaboración propia a partir de datos de los censos de 2001 (arriba) y 2011 (abajo). 
Figura 3. Barrializaciones superpuestas: vivienda de más de $120 \mathrm{~m}^{2}$, ocupación alta, formación universitaria y nacionalidad extranjera (Madrid)
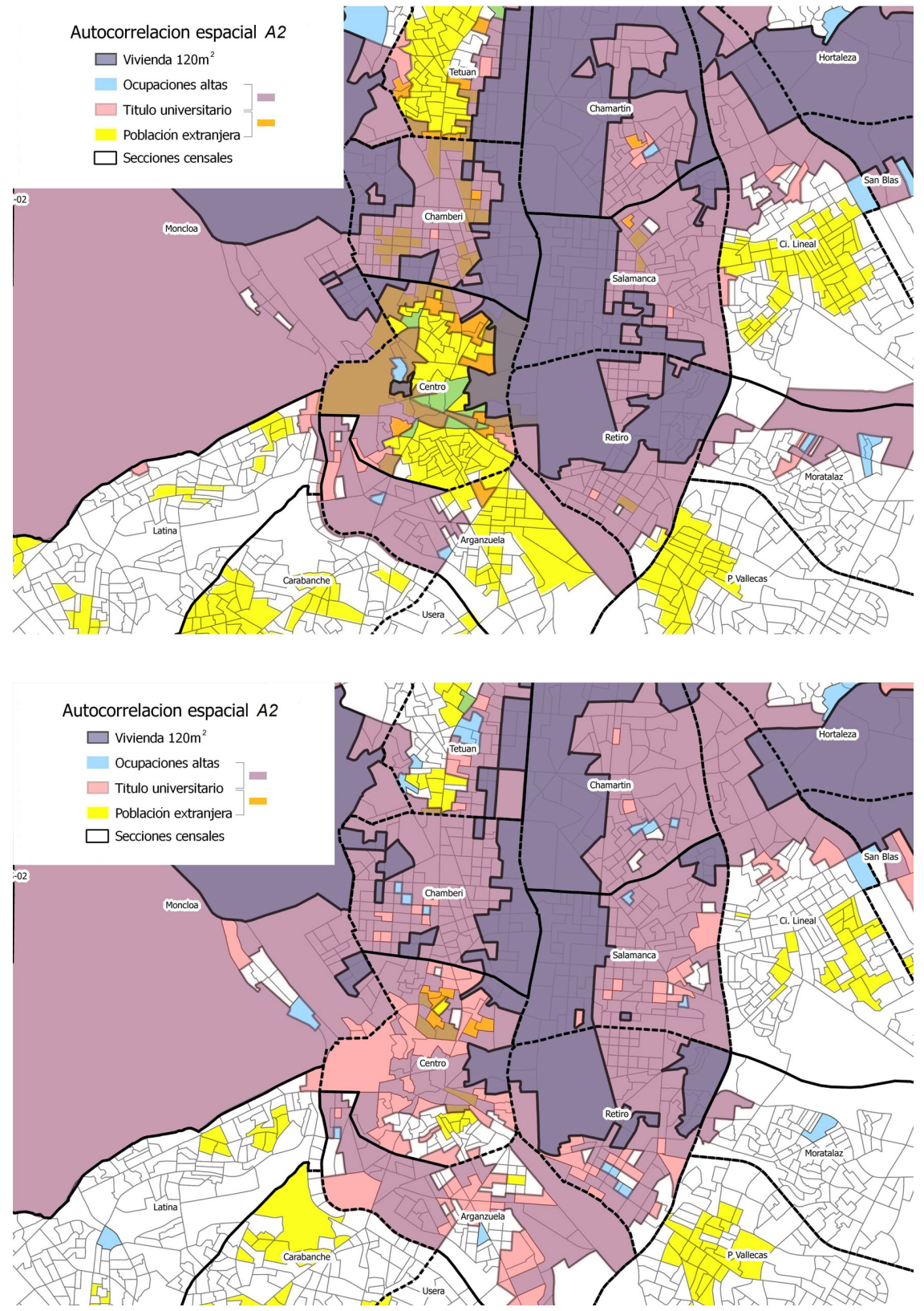

Fuente: elaboración propia a partir de datos de los censos de 2001 (arriba) y 2011 (abajo). 
ocupaciones de categoría alta se localiza dentro de las zonas de concentración y agrupación de la población con estudios universitarios. Sin embargo, en 2001 la coincidencia entre ambas es casi perfecta y aparece dominando el mapa con colores lila claro. La correlación entre ocupaciones altas y estudios universitarios era de 0,97 en 2001 y de 0,87 en 2011. Es decir, se ha producido una mayor extensión de barrialización de población con título universitario en Sant Antoni, Gòtic, Poble Nou y Fort Pienc (Barcelona), así como en los distritos de Centro, Arganzuela y en la zona sur de Retiro. Así, la presencia de población con título universitario ha reducido su capacidad como marcador de clase alta.

\subsubsection{Población residente en viviendas de más de $120 \mathrm{~m}^{2}$}

Finalmente, la concentración y la agrupación de población residente en viviendas de más de $120 \mathrm{~m}^{2}$ es la zona más restringida. Se localiza en el centro de las otras barrializaciones, en Sarriá, Sant Gervasi, Pedralbes, las Tres Torres y la Dreta de l'Eixample (Barcelona) o en los distritos de Chamartín, Salamanca, Retiro y Moncloa (Madrid). Este núcleo más reducido (social y territorialmente) cuenta con patrimonio consolidado y está alejado tanto de la población sin características de clase alta (capital cultural o rentas altas) como de la población extranjera. Como se vio anteriormente, además de su mayor separación espacial de los grupos desfavorecidos, la barrialización de población residente en viviendas de más de $120 \mathrm{~m}^{2}$ coincide con las zonas de mayores porcentajes de población ocupada en categorías altas (valores que duplican la media). Como corolario, las zonas en las que se localiza la población con más patrimonio son las que muestran los mayores niveles de ocupación en categorías altas y de población con estudios universitarios.

\subsubsection{Población extranjera}

Los tres elementos más destacados en la barrialización de la población extranjera son: su localización apartada de los sectores propios de la clase alta, su asociación a zonas más desfavorecidas y su importante reducción en los distritos centrales del 2001 al 2011.

La población de nacionalidad extranjera presenta dificultades añadidas de cara a su estudio: muestra una fuerte heterogeneidad interna, con sesgos marcados por nacionalidades. Los ciudadanos nacionales de países con importantes rentas per cápita (como los miembros de la UE) presentan patrones residenciales similares a los de las clases medias y altas, y los demás nacionales presentan rasgos más populares. Además, del 2001 al 2011, la población extranjera ha incrementado fuertemente su valor absoluto y ha sufrido importantes transformaciones en su composición.

A escala metropolitana, si comparáramos las barrializaciones (A2) de población extranjera, observaríamos que en 2001 tenía un peso importante en partes de la ciudad central, barrios y centros históricos, así como también en municipios retirados hacia la segunda corona. En 2011 esta barrialización reduce fuertemente su presencia en los centros urbanos y en los municipios metropolitanos más residenciales. En 2011, las nuevas zonas de barrialización de población 
extranjera tienden a alejarse del centro y a concentrarse en municipios con actividad industrial y logística, zonas populares de la primera corona y municipios populares aún más alejados. El cambio no debe leerse solo en términos de desplazamiento de la población extranjera. Se da la (aparente) paradoja de que todas las secciones censales que dejan de estar incluidas en la barrialización (A2) de la población extranjera han incrementado sus efectivos entre 2001 y 2011. Lo que ocurre es que este incremento no ha sido tan rápido como el producido en las nuevas secciones que sí están incluidas en la nueva barrialización concentrada de población de nacionalidad extranjera (A2) en 2011. Es posible que en un periodo de flujo constante las poblaciones extranjeras buscaran intencionalmente diversificar sus zonas de residencia y, también, que los cambios en expectativas y precios de las zonas centrales hayan reforzado aún más esta diversificación hasta hacer de algunas periferias metropolitanas las nuevas zonas de especialización residencial de la población extranjera.

\section{Conclusiones}

Los fuertes cambios sociales y poblacionales que han tenido lugar durante la primera década del siglo XXI enmarcan también importantes transformaciones socioterritoriales de las grandes metrópolis españolas.

En primer lugar, la desconcentración. A pesar de incluir un inicio de crisis y de recentralización poblacional, la primera década del siglo xxi aún se salda con movimientos de desconcentración (Bayona y Pujadas, 2019). Como hemos visto, la población aumenta su distancia media entre miembros en un proceso generalizado de casi un 5\%. Esto afecta especialmente a la población extranjera, pero menos a la población con estudios universitarios, que tiende a igualarse y a concentrarse en las capitales metropolitanas haciendo más diferentes los barrios de estas grandes ciudades del resto del territorio metropolitano.

También hay que destacar la pérdida de importancia relativa de clases populares (nacionales y extranjeras) en zonas muy claras y localizadas del centro: en Tetuán y Centro, en el caso de Madrid; así como en los barrios de Gòtic, Sant Pere, Santa Caterina i la Ribera y Glòries, en el caso de Barcelona. La evidencia cuantitativa de esta transformación de barrios puede y necesita ser complementada con estudios de caso que relacionen estos cambios poblacionales con las dinámicas de precios del mercado de alquiler y vivienda, por un lado (como hacen Fernández y Roch, 2012), y con diferentes políticas urbanas: de vivienda (Jiménez Romera y Fernández Ramírez, 2014) o de cultura, turismo y medio ambiente (García Pérez, 2014), por otro. De forma complementaria, sería necesario extender estos estudios de caso demográficos, inmobiliarios y de política pública en las nuevas zonas de periferia donde se han incrustado nuevos fragmentos de concentraciones de población desfavorecida (nacional y/o extranjera).

En definitiva, si hubiera que sintetizar el conjunto de las dinámicas, los grupos y las transformaciones, el periodo 2001-2011 asiste a un aumento de la concentración y agrupación de las zonas de clase alta, que contrasta con la 
mayor segregación, pero también mayor fragmentación de los barrios populares. Podemos etiquetar el proceso como una polarización desigual: por un lado, zonas de clase alta que se expanden, se agrupan y se reconcentran más, y, por otro, zonas populares que también se expanden y se concentran, pero de forma más fragmentada. Sea por desplazamiento o transformación de la estructura o, más probablemente, por una combinación de estos dos procesos, el proceso de elitización y gentrificación de las capitales resulta evidente, así como la extensión en ellas de la población (nacional o extranjera) con estudios universitarios.

Finalmente, el análisis del periodo 2001-2011 apunta a una fuerte elitización de las capitales y sus centros. Estos resultados son coherentes con los obtenidos por López-Gay (2018), que muestra también su profundización a partir de 2011 para Barcelona. Pero son tendencias que hemos visto replicarse y continuar en muchas otras capitales europeas como Ámsterdam y Róterdam (Hochstenbach y Musterd, 2018) o Tallin, Estocolmo, Helsinki, Oslo, Atenas, Budapest, Madrid y Milán (Tammaru et al., 2018). En Barcelona y Madrid hemos visto como se produce un proceso que podría resumirse en la fragmentación espacial de las zonas populares que contrasta con el aumento en extensión, agrupación y homogeneidad de las zonas más elitizadas. Es posible que entre 2001 y 2011 diferentes niveles de la Administración hayan podido estar dando más importancia a desconcentrar las zonas de población desfavorecida que a corregir desigualdades estructurales, a colaborar en la dignificación de estas zonas o a garantizar en ellas el acceso a la vivienda para sus vecinos.

Esto aparece de forma especialmente clara en los centros: una dinámica de elitización y fraccionamiento de los barrios populares. Esta progresiva elitización es un problema que va más allá del debate sobre gentrificación con o sin desplazamiento poblacional. El centro tiene una fuerte capacidad de visibilización colectiva. La presencia de concentraciones de grupos desfavorecidos en los centros de las grandes capitales dota a estos grupos de existencia social en el imaginario colectivo (Rubiales, 2017b). Una presencia positiva, central y de calidad supone una de las herramientas más potentes de desestigmatización 7 . Por eso las políticas urbanas deberían incorporar como objetivo mantener en nuestros centros una presencia popular y diversa, con buenos niveles de calidad urbana, de equipamientos públicos y con posibilidad de acceso a la vivienda.

Merece la pena acabar con un ejercicio reflexivo sobre la responsabilidad que también tenemos desde la investigación y la producción de conocimiento. Quizás no hemos conseguido en este tiempo ampliar nuestras cuantificaciones más allá de los cálculos de segregación. Si lo hemos hecho poco hasta ahora, de cara al futuro inmediato deberíamos plantearnos la generación de cuantificaciones sobre otros aspectos que también inciden en la vulnerabilidad: la igualdad, el nivel de cobertura de diferentes necesidades sociales, los niveles de

7. Un ejemplo paradigmático ha sido el caso del colectivo LGTB en Chueca. Boivin (2011) documenta de forma brillante cómo esta agrupación territorial fue un movimiento voluntario y consciente, que tenía como objetivo explícito luchar contra la creciente estigmatización que estaba sufriendo y que resultó una estrategia exitosa. 
estigmatización territorial, etc. Porque si el único problema que planteamos en el análisis territorial de las desigualdades de nuestras ciudades es la segregación de las poblaciones vulnerables, la única respuesta política al problema será la desconcentración. Venga esta bajo el nombre de mezcla social, gentrificación, integración o desplazamiento a las terceras coronas de las personas vulnerables entre las vulnerables.

\section{Referencias bibliográficas}

ArbaCI, S. y RAE, I. (2014). «Efecto barrio y desigualdades: Evidencias para desmitificar las políticas urbanas de diversificación residencial». ACE: Architecture, City and Environment, 9 (26), 147-176. $<$ http://dx.doi.org/10.5821/ace.9.26.3687>

Andersson, E.K. y Malmberg, B. (2015). "Contextual effects on educational attainment in individualised, scalable neighbourhoods: Differences across gender and social class». Urban Studies, 52 (12), 2117-2133. $<$ http://dx.doi.org/10.1177/0042098014542487>

Anselin, L. (1995). «Local indicators of spatial association-LISA». Geographical Analysis, 27 (2), 93-115. <https://doi.org/10.1111/j.1538-4632.1995.tb00338.x>

Anselin, L.; Syabri, I. y Kho, Y. (2006). «GeoDa: An introduction to spatial data analysis». Geographical Analysis, 38 (1), 5-22. <http://dx.doi.org/10.1111/j.0016-7363.2005.00671.x>

Bayona, J. y Blanco Cardona, M.J. (2004). «Pautes d'associació en l'espai de la població de nacionalitat estrangera a Barcelona». Documents d'Anàlisi Geogràfica, $43,107-122$.

Bayona i Carrasco, J. y Gil-Alonso, F. (2012). «Suburbanisation and international immigration: The case of the Barcelona metropolitan region (1998-2009)». Voor Economische, 3 (3), 312-329. $<$ https://doi.org/10.1111/j.1467-9663.2011.00687.x>

Bayona-I-Carrasco, J. y Pujadas, I. (2019). «Las grandes áreas metropolitanas en España: Del crecimiento y la expansión residencial al estancamiento poblacional». Documents d'Anàlisi Geogràfica, 66 (1), 27-55. $<$ https://doi.org/10.5565/rev/dag.576>

Blokland, T.; Hentschel, C.; Holm, A.; Lebuhn, H. y Margalit, T. (2015). «Urban citizenship and right to the city: The fragmentation of claims». International Journal of Urban and Regional Research, 39 (4), 655-665. $<$ http://dx.doi.org/10.1111/1468-2427.12259>

Borvin, Renaud René (2011). «De la ambigüedad del clóset a la cultura del gueto gay: Género y homosexualidad en París, Madrid y México». La Ventana: Revista de Estudios de Género, 34, 146-190. Recuperado de <http://www.scielo.org.mx/scielo.php?script=sci_arttext\&pid=S1405-94362011000200007\&lng=es\&tlng=en>.

Champion, T. (2001). Urbanization, Suburbanization, Counterurbanization and Reurbanization: In Handbook of Urban Studies. Londres: SAGE Publications Ltd., 143-161. $<$ http://dx.doi.org/10.4135/9781848608375.n9>

Clark, W.A.; Anderson, E.; Östh, J. y Malmberg, B. (2015). «A multiscalar analysis of neighborhood composition in Los Angeles, 2000-2010: A location-based 
approach to segregation and diversity». Annals of the Association of American Geographers, 105 (6), 1260-1284. <http://dx.doi.org/10.1080/00045608.2015.1072790>

Colectivo IOÉ (2012). Impactos de la crisis sobre la población inmigrante. Madrid: Organización Internacional para las Migraciones.

Coll Noguera, M. y Pujadas, I. (2007). «Migració i segregació residencial a la Regió Metropolitana de Barcelona: Sant Cugat del Vallès com a elecció residencial per a les categories professionals altes». Treballs de la Societat Catalana de Geografia, 67, 35-50.

Cox, K.R. (1969). «The Voting Decision in a Spatial Context». Progress in Geography, $1,81-117$.

DAwKINS, C.J. (2004). «Measuring the spatial pattern of residential segregation». Urban Studies, 41 (4), 833-851. <https://doi.org/10.1080/0042098042000194133>

Domingo i Valls, A. (2014). Catalunya al mirall de la immigració: Demografia $i$ identitat nacional. Barcelona: L'Avenç, 355.

Esteve, A. (2003). El Nomenclàtor com a font per a l'estudi territorial de la població a Catalunya: Aplicacions 1857-1998. Departament de Geografia de la UAB i Centre d'Estudis Demogràfics. Tesi doctoral.

Fernández, C. y Roch, F. (2012). «La quiebra de la ciudad global y sus efectos en la morfología urbana: Madrid, bajo la lógica inmobiliaria de la acumulacióndesposesión». Urban, 3, 45-63. Recuperado de <http://polired.upm.es/index.php/ urban/article/view/1807>.

Folch, D. C. y Rey, S. J. (2016). «The centralization index: A measure of local spatial segregation». Papers in Regional Science, 95 (3), 555-576.

FranK, A.I. (2003). Using measures of spatial autocorrelation to describe socio-economic and racial residential patterns in US urban areas, 13, 287. Nueva York: Taylor \& Francis.

García Pérez, E. (2014). «Gentrificación en Madrid: De la burbuja a la crisis». Revista de Geografia Norte Grande, 58, 71-91. <https://dx.doi.org/10.4067/S0718-34022014000200005>

Harvey, D. (1998). La condición de la posmodernidad. Buenos Aires: Amorrortu.

- (2008). «El derecho a la ciudad». New Left Review, 53 (4), 23-39.

Hochstendach, C. y Musterd, S. (2018). "Gentrification and the suburbanization of poverty: Changing urban geographies through boom and bust periods». Urban Geography, 39 (1), 26-53. $<$ https://doi.org/10.1080/02723638.2016.1276718>

Huckfeldt, R.; Sprague, J. y Levine, J. (2000). «Dynamics of Collective Deliberation in the 1996 Election: Campaign Effects on Accessibility, Certainty, and Accuracy». American Political Science Review, 94 (3), 641-651. $<$ http://dx.doi.org/10.2307/2585836>

Israel, E. y Frenkel, A. (2018). «Social justice and spatial inequality: Toward a conceptual framework». Progress in Human Geography, 42 (5), 647-665. <https://doi.org/10.1177/0309132517702969>

Jiménez Romera, C. y Fernández Ramírez, C. (2014). «Casas sin gente, gente sin casas: El fracaso del modelo inmobiliario español». Revista INVI, 29 (82), 133-155. $<$ https://dx.doi.org/10.4067/S0718-83582014000300005>

Knies, G.; Nandi, A. y Platt, L. (2016). «Life satisfaction, ethnicity and neighbourhoods: Is there an effect of neighbourhood ethnic composition on life satisfaction?». Social Science Research, 60, 110-124. $<$ http://dx.doi.org/10.1016/j.ssresearch.2016.01.010> 
Koch, A. y Mandl, P. (eds.) (2011). Modeling and simulating urban processes. Münster: LIT Verlag.

Lefebvre, H. (1976). Espacio y política: El derecho a la ciudad, II. Barcelona: Península.

Lloyd, C.D. y ShutTleworth, I.G. (eds.) (2014). Social-spatial segregation: Concepts, processes and outcomes. Bristol, UK: Policy Press. <https://doi.org/10.1332/policypress/9781447301356.001.0001>

López-GaY, A. (2018). "Cambio en la composición social y gentrificación en Barcelona: Una mirada a través de los flujos migratorios y residenciales». Papers: Regió Metropolitana de Barcelona: Territori, estratègies, planejament, 60, 80-93.

Manley, D.; Jones, K. y Johnston, R. (2019). «Multiscale Segregation: Multilevel Modeling of Dissimilarity: Challenging the Stylized Fact That Segregation Is Greater the Finer the Spatial Scale». The Professional Geographer, 1-13.

Martori, J.C. y Hoberg, K. (2008). «Nuevas técnicas de estadística espacial para la detección de clusters residenciales de población inmigrante». Scripta Nova: Revista Electrónica de Geografía y Ciencias Sociales, 12 (263).

Miller, W.L. (1977). Electoral Dynamics. Palgrave: Macmillan, 242 p. <http://dx.doi.org/10.1007/978-1-349-15851-5_7>

Moran, P. (1948). «The interpretation of statistical maps». Journal of the Royal Statistical Society, 10 (2), 243-251.

Moreno, A.; Fernández, F.; VÁzquez, C. y Prieto, M.E. (2003). La distribución espacial de la renta en la Comunidad de Madrid: análisis y aplicaciones. Madrid: Instituto de Estadística de la Comunidad de Madrid.

Pujadas, I. (2009). «Movilidad residencial y expansión urbana en la Región Metropolitana de Barcelona, 1982-2005». Scripta Nova: Revista Electrónica de Geografía y Ciencias Sociales, 13 (290).

Rocha, F. y Aragón, J. (2012). «La crisis económica y sus efectos sobre el empleo en España». Gaceta Sindical, 19, 67-90.

Rubiales, M. (2016). «Territorio y crisis: Impacto territorial de la crisis económica en las regiones de Madrid y Barcelona». Scripta Nova: Revista Electrónica de Ciencias Sociales, XX, 459-567.

- (2017a). Patrones socioterritoriales de las clases altas en las regiones metropolitanas de Barcelona y Madrid (2001-2015). Barcelona: Universitat de Barcelona. Tesis doctoral.

- (2017b). «Nueva estructura de clases, una mirada territorial». Encrucijadas: Revista Critica de Ciencias Sociales, 14, 1402.

Rubiales. R.; Bayona, J. y Pujadas, I. (2012). «Patrones espaciales de la segregación residencial en la Región Metropolitana de Barcelona: Pautas de segregación de los grupos altos». Scripta Nova: Revista Electrónica de Geografía y Ciencias Sociales, 16.

- (2013). «Distribución espacial de las clases altas en la región metropolitana de Madrid 2001-2011». Anales de Geografía, 33 (2), 107-136. <https://doi.org/10.5209/rev_aguc.2013.v33.n2.43003>

SAmpson, R.J. (2019). "Neighbourhood effects and beyond: Explaining the paradoxes of inequality in the changing American metropolis». Urban Studies, 56 (1), 3-32. <http://dx.doi.org/10.1177/0042098018795363>

Sharkey, P. y FABER, J.W. (2014). "Where, when, why, and for whom do residential contexts matter?: Moving away from the dichotomous understanding of neighborhood effects». Annual Review of Sociology, 40, 559-579. <http://dx.doi.org/10.1146/annurev-soc-071913-043350> 
Sienkiewicz, J.J.; Hapke, P. y Faist, T. (2016). «Social Comparisons in Migration and Inequality Studies: A Literature Review and Evidence from a Pilot Study». En: From Heterogeneities to Inequalities. Bielefeld: DFG Research Center (SFB), 882.

Sorando, D. y Leal, J. (2019). «Distantes y desiguales: El declive de la mezcla social en Barcelona y Madrid». REIS: Revista Española de Investigación Sociológica, 167, 125-148.

Tammaru, T.; Van Ham, M.; Marcinçzak, S. y Musterd, S. (2017). «Socioeconomic segregation in European capital cities. Increasing separation between poor and rich». Urban Geography, 38 (7), 1062-1083.

Tammaru, T.; Marcinczak, S.; Aunap, R.; Van Ham, M. y Janssen, H. (2018). «Relationship between income inequality and residential segregation of socioeconomic groups». Regional Studies, 1-12. <http://dx.doi.org/10.1080/00343404.2018.1540035>

Tapada-Berteli, T. y Arbaci, S. (2011). «Proyectos de regeneración urbana en Barcelona contra la segregación socioespacial (1986-2009): ¿ Solución o mito?». ACE: Architecture, City and Environment, 6 (17), 187-222. <https://doi.org/10.5821/ace.v6i17.2534>

Wacquant, L.; Slater, T. y Pereira, V.B. (2014). "Territorial stigmatization in action». Environment and Planning A, 46 (6), 1270-1280. $<$ https://doi.org/10.1068/a4606ge> 
